\title{
PHARMACOGENOMICS AND REGULATION OF APOPTOSIS IN ACUTE MYELOID LEUKAEMIA
}

\section{FARMAKOGENOMIKA I REGULACIJA APOPTOZE U AKUTNOJ MIJELOIDNOJ LEUKEMIJI}

\author{
Zlatko Pravdić ${ }^{1,2}$, Nada Suvajdžić Vuković ${ }^{1,2}$
}

${ }^{1}$ University of Belgrade, Faculty of Medicine, Belgrade, Serbia

${ }^{2}$ University Clinical Centre of Serbia, Clinic for Hematology, Belgrade, Serbia

Correspondence: zlatko.pravdic@gmail.com

\section{Abstract}

Acute myeloid leukaemia (AML) is a heterogenous clonal hematopoietic malignancy primarily treated with combination of cytarabine (ara-C) and anthracyclines. Despite high

Keywords:

AML,

pharmacogenomics,

ara-C,

anthracyclines,

apoptosis remission rates, especially in younger patients, a vast majority of patients die due to relapse or chemotherapy/stem cell transplantation-related toxicity. The partial explanation for this grim clinical outcome lies in the patients' genetic variability. In this review, we will summarize how genetic polymorphisms of proteins, in metabolic paths of cytarabine and anthracyclines and proteins involved in regulation of apoptosis, influence efficacy and toxicity in the AML treatment.

\section{Sažetak}

Akutna mijeloidna leukemija (AML) je klonalna bolest hematopoeze, heterogena kako u etiopatogenetskom, tako i u smislu ishoda lečenja. Osnov terapije predstavlja kombinacija ci-

Ključne reči:

AML, farmakogenomika, ara-C, antraciklini, apoptoza tarabina (ara-C) i antraciklina. Uprkos visokoj stopi remisija, posebno kod mlađih, većina bolesnika umire zbog relapsa ili usled komplikacija izazvanih hemioterapijom/transplantacijom matične ćelije hematopoeze. Nepovoljna prognoza AML se jednim delom može objasniti genetskom varijabilnošću bolesnika. Cilj ovog preglednog članka je prikaz uticaja genetskih polimorfizama metaboličkih puteva citarabina i antraciklina, kao i proteina uključenih u regulaciju apoptoze na efikasnost i toksičnost lečenja AML.
Pravdić Z. et al. MedPodml 2020, 71(4):10-16

(c)

The authors declare no conflicts of interest. doi:10.5937/mp71-28140

Editorial board: podmladak.med.bg@gmail.com 


\section{Introduction}

Acute myeloid leukaemia (AML) is a heterogenous clonal hematopoietic malignancy that arises from clonal expansion either of stem or early progenitor hematopoietic cells (granulocyte, erythrocyte or megakaryocyte) (1). Numerous genetic and epigenetic changes in immature cells (blasts) blocks them in their differentiation and lead to their accumulation in bone marrow. Leukemic blasts interfere with growth of normal blood cells producing anaemia, infection and bleeding (2). Acute myeloid leukaemia, with incidence from 5 to 8 per 100000 per year in European population, is the most prevalent adult acute leukaemia (2). Moreover, $65 \%$ of patients are older than 60 years. Incidence and mortality rise with age, with 67 years as median age at diagnosis (3).

Diagnosis of AML is based on bone marrow aspiration analysis. More than $\geq 20 \%$ of marrow blasts is diagnostic, except for AML with recurrent cytogenetic abnormalities: $\operatorname{inv}(16)$ or $\mathrm{t}(16,16), \mathrm{t}(8 ; 21)$ or $\mathrm{t}(15 ; 17)$ (2). Multiparameter flow cytometry is recommended for precise diagnosis. Cytogenetic abnormalities are present in $55 \%$ of AML patients (4). Additional point mutations and small insertions/deletions are detected in $85 \%$ of patients with normal cytogenetics, using advanced molecular techniques (4). Conventional metaphase cytogenetic analysis and molecular genetic testing are mandatory in diagnosis since they define specific AML entities and are the best pre-treatment prognosticators $(2,4)$. Karyotype and molecular parameters stratify AML patients into: favourable, intermediate and adverse risk group (2). The likelihood of chemotherapy-alone cure rate is approximately $65-70 \%$ with favourable risk group. In intermediate risk group, long-term survival rate is 30 $40 \%$, while in adverse risk group it is $<5-10 \%$, without allogenic stem cell transplantation (SCT) (5).

The patient with AML caries two genomes: constitutional (germline), that is present in every cell except in leukemic cells and leukemic genome (somatic), which harbours somatic mutations that lead to leukemogenesis that evolves in the course of the disease (6). It is postulated that genetic variants i.e. polymorphisms in germline genome are affecting drug transport and metabolism, along with drug efficacy and occurrence of adverse events. However, genetic variants in somatic genome are responsible for chemoresistance (6).

Standard of care in AML in last four decades, except for acute promyelocytic leukaemia, has been a combination of nucleoside analogue cytarabine (ara-C) and anthracycline (daunorubicin or idarubicin) known as " $7+3$ " induction regimen. Complete remission (CR) rate in adults ( $<60$ years) is $60-80 \%$, while $40-60 \%$ for older than 60 years. Post-remission treatment is based on three cycles of intermediary doses of ara-C, and allogenic SCT in eligible patients (2). Despite these CR rates, about half of the patients experience relapse, primarily due to chemotherapy failure to eliminate minimal residual disease (7). Finally, the majority of patients die due to chemotherapy/SCT-related toxicity, primarily resistant disease or relapse, with the 5 -year overall survival (OS) $<50 \%$ in $<60$ years and $<20 \%$ for older $(2,4,7)$.

\section{Ara-C}

Ara-C is a backbone of AML therapy. It is a deoxycytidine nucleoside analogue $(5,8)$, and in its activated triphosphate form (ara-CTP) it competes with deoxycytidine triphosphate (dCTP) for incorporation into DNA. Besides, ara-C inhibits both conversion of cytidine triphosphate (CTP) to dCTP and DNA and RNA polymerases, all essential for DNA synthesis (5).

\section{Transport and metabolism of ara-C}

Ara-C enters leukemic cells by nucleoside transporters. Primary transporter, that carries $80 \%$ of the drug, is SLC29A1, also known as hENT1 (solute carrier family 29 member 1 (human equilibrative nucleoside transporter 1)), $(7,8)$ - Figure 1. After entering cell via SLC29A1, ara-C activation by 5 ' phosphorylation is conducted by kinases. First step is conversion to ara-C monophosphate (ara-CMP), conducted by deoxycytidine kinase (DCK), then to ara-C diphosphate, mediated by deoxycytidylate kinase or CMPK (cytidine monophosphate kinase), and finally to active nucleoside analogue ara-CTP, by nucleoside diphosphate kinase (NDPK). It is of note that therapeutic effect is directly influenced by intracellular concentration of ara-CTP (5). Ara-C is inactivated either by removing of amino group by deaminases or by dephosphorylation (back to ara-C) by 5 'nucleotidases (5). Crucial indicators for sensitivity/resistance to ara-C are: activation via kinases, inhibition by deaminases, half-life and intracellular concentration of ara-CTP and finally, the extent of DNA incorporation (5).

\section{Polymorphisms of main genes involved in the ara- $\mathrm{C}$ metabolism and transport}

\section{SLC29A1 - main drug transporter in standard ara-C} doses

Plasma concentrations $>10 \mu \mathrm{M}$, achieved by high doses of ara-C $\left(2-3 \mathrm{~g} / \mathrm{m}^{2}\right)$ freely diffuse into leukemic cells. However, in plasma concentrations $<1 \mu \mathrm{M}$, that are reached by induction chemotherapy " $7+3$ " (100-200 mg/m²), transport of ara-C into leukemic blasts is highly reliant on SLC29A1 (5,8). Addressing this issue, SLC29A1 gene, located on chromosome 6q21.1-21.2, shows low mutability compared to other transporters (8). Shorter disease-free survival (DFS) and shorter OS was observed in AML patients lacking SLC29A1 $(5,7,8)$. The first studied genetic variants of SLC29A1 gene - four synonymous and two nonsynonymous, had no impact on nucleoside uptake $(7,8)$. However, low activity SLC29A1 variants rs 324148 and rs9394992 individually or together were associated 


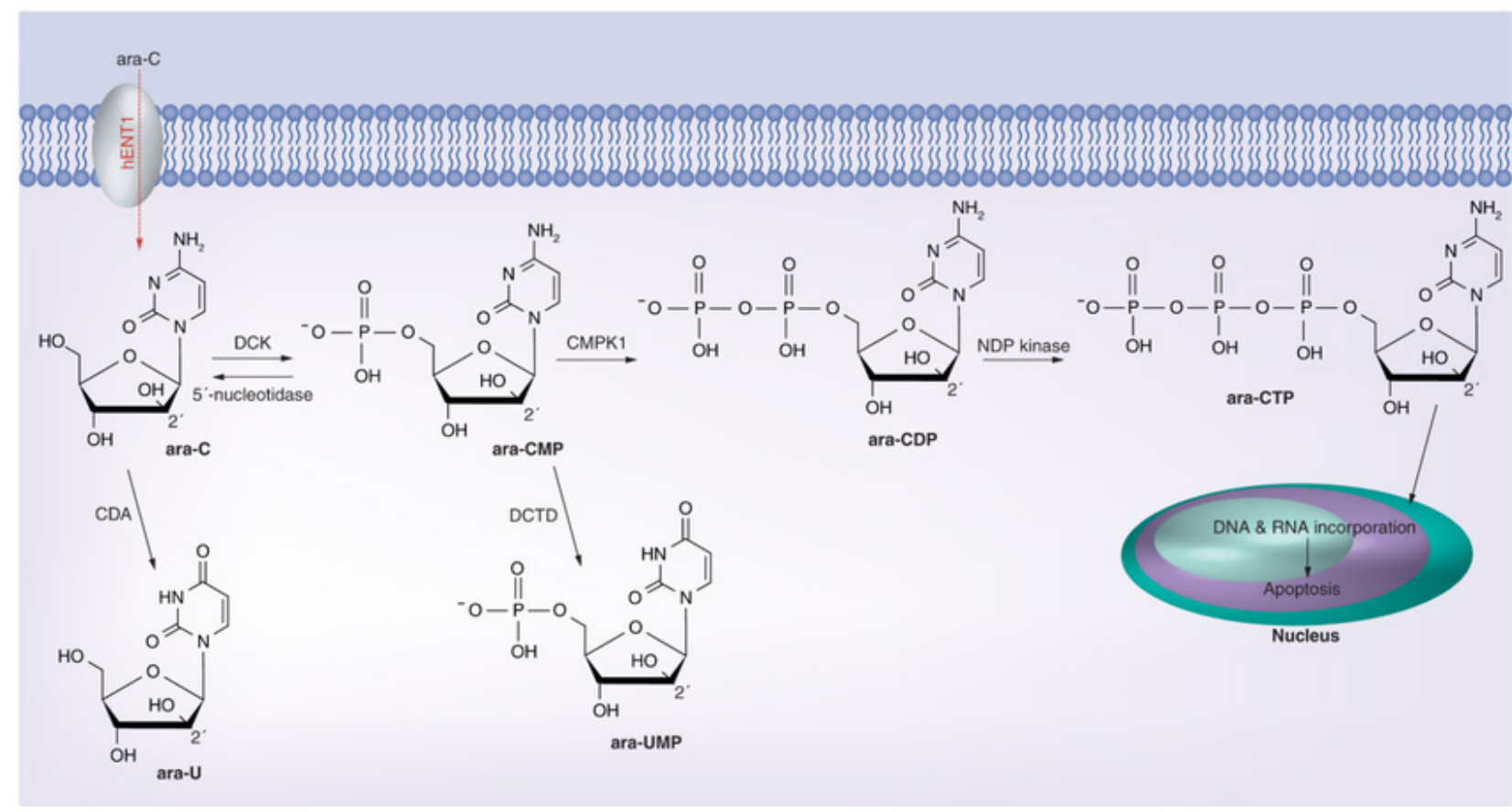

Figure 1. Ara-C metabolism (adapted from Emadi et al).

Abbreviations: ara-C (cytarabine), ara-CMP (ara-C monophosphate), ara-CDP (ara-C diphosphate), ara-CTP (ara-C triphosphate), ara-U (ara-Uracil), ara-UMP (ara-U monophosphate), hENT1 (human equlibrative nucleoside transporter 1), DCK (deoxycytidine kinase), CMPK1 (cytidine monophosphate kinase), NDP (nucleoside diphosphate) kinase

with lower DFS, OS, mRNA expression and increased rate of relapse (RR) (9). In addition, transcription regulators such as PPAR $\alpha$ or HIF- $\alpha$ can affect SLC29A1 expression and thus impact intracellular ara-C levels $(5,8)$. Considering ara-C a cornerstone of AML treatment, SLC29A1 polymorphisms can be presumed as predictive biomarkers in AML (5).

The kinases - ara-C activators

Deoxycytidine kinase performs first, rate limiting, phosphorylation step of ara-C activation. The DCK gene resides on 4q13.3-q21 chromosome. Some ara-C resistant cell lines show loss of DCK activity (8). Longer event-free survival (EFS) was observed in AML patients with higher DCK mRNA expression in leukemic blasts. Conversely, enzymatic activity was not correlated with mRNA expression $(5,7,8)$. Wild-type single nucleotide polymorphisms (SNP) $(201 \mathrm{C}>\mathrm{T}$ and $360 \mathrm{G}>\mathrm{C}$ ) in $D C K$ promoter regions, were associated with decreased mRNA expression, lower enzymatic activity and inferior 2-year EFS. Variant 360G $>C$ in Asian population had no impact on either CR or survival, while in Hispanic population both variants were associated with higher OS and lower in vitro resistance $(5,7)$. Variant of $D C K$, rs4694362 is associated with higher OS, while rs72552079 and rs11543896 variants were associated with higher CR rate (7). Moreover, major polymorphisms in CMPK and NDPK genes have not been identified $(5,7)$, while expression levels of $N M E 1$ gene (NDPK encoding gene) have been reported to be prognostic in various tumours, including AML. That is, higher NME1 expression is related with chemoresistance (including ara-C) and lower OS in AML patients (7).

\section{Ara-C inhibition}

The 5' nucleotidases (NT5C) are enzymes that dephosphorylate various nucleotides and nucleotide analogues, thus maintaining balanced nucleotide pools in cells $(7,8)$. The function of cytosolic 5 ' nucleotidase II (NT5C2) is opposed to DCK. Namely, its increased expression was associated with resistance to ara-C (and other nucleoside analogues) and lower OS in AML patients $(5,7)$. Additionally, expression of NT5C2 reflects proliferative activity in blasts and can be used as parameter of leukaemia aggressiveness (8). Considering this matter, 42 SNP were identified, three of them nonsynonymous, with no effect on NT5C activity (7).

Cytidine deaminase (CDA) is the cardinal deactivating enzyme of ara-C (7). Its overexpression has been associated with resistance to ara- $\mathrm{C}$ and relapse in AML patients. Conversely, lower CDA concentrations were related to prolonged remission $(5,7)$. Genetic evaluation of $C D A$ revealed three nonsynonymous SNPs $435 \mathrm{~T}>\mathrm{C}, 208 \mathrm{G}>\mathrm{A}$ and $79 \mathrm{~A}>\mathrm{C}$ resulting in reduced enzyme activity. The 79A $>\mathrm{C}$ (rs207261) variant allele was corelated with inferior CR rate and shorter OS, particularly in AML with FLT3-ITD mutation AML, along with increased occurrence of mucositis and treatment-related mortality (TRM) (7). Moreover, CDA polymorphisms 33delC, $451 \mathrm{C}>\mathrm{T}$ and $435 \mathrm{C}>\mathrm{T}$ are associated with decreased CR rate and lesser grade $\geq 3$ infections, while $451 \mathrm{C}>\mathrm{T}$ minor allele was associated with poorer OS (7). The aforementioned inactivating $C D A$ variants could lead to increased risk of toxicity and consequent 
lower OS (7). Polymorphisms in other deaminating enzyme deoxycytidylate deaminase (DCTD), a dCMP deaminase, are of uncertain effect in AML patients $(5,7,8)$. Poorer OS has been observed when SNPs of DCTD minor allele (rs17331744) and CDA wild-type (rs10916827) were present alongside (7), being both cardinal deaminating enzymes of ara-C.

Additional regulator of intracellular ara-C concentration, and its effect, is intracellular level of CTP and dCTP nucleotides. Leukemic cells of resistant AML patients harbour high levels of dCTP (5), which regulates ara-C effect by: DCK inactivation, CDA activation and by competing with ara-CTP for DNA incorporation (8). The concentration of CTP/dCTP nucleotides is controlled by both ribonucleotide reductase (RRM) and cytidine-5'-triphosphate synthetase (CTPS1) (7). Activity of RRM enzyme is correlated with chemosensitivity/resistance to ara-C (5). Variant alleles in RRM subunits: RRM1 (rs2268166, rs11031136, rs7130539 and rs4593998) and $R R M 2 B$ (rs2607662 and rs2853229) are connected with decreased DFS in Caucasians (5). In contrast, at least 24 SNPs in CTPS1 gene showed no significant associations in AML patients (5).

\section{Anthracyclines}

Anthracyclines are another important chemotherapeutic class utilized in AML. Clinically the most important anthracyclines are: daunorubicin or daunoblastin (DA) and its analogues: idarubicin, doxorubicin and epirubicin (5). Anthracyclines have three major mechanism of action: intercalation between DNA base pairs with consecutive DNA/ RNA synthesis disruption, topoisomerase II inhibition and consecutive formation of double-strand DNA brakes during replication and by formation of reactive oxygen species (ROS) $(5,7)$. The most common used anthracyclines in " $7+3$ " regimen are daunorubicin and idarubicin.

\section{Anthracycline membrane transporters}

Anthracycline intracellular transport is the most clinically significant pharmacogenomic factor. Namely, ATPbinding cassette $(A B C)$ family genes encode membrane efflux pumps, which are important in anthracycline transport and drug resistance. Three $\mathrm{ABC}$ transporters most accountable for the multidrug resistance (MDR) in humans are: P-glycoprotein (P-gp) (ABCB1, MDR1), MRP1 (ABCC1) and BCRP (ABCG2, ABCP, MXR) (5). The expression and function of $A B C B 1$ in AML patients is associated to chemoresistance and CR. Expression of $A B C B 1$ and anthracycline efflux rise with patient's age (5). Most evaluated polymorphisms of $A B C B 1$ are: $1236 \mathrm{C}>\mathrm{T}$ (rs1128503), 2677G $>\mathrm{A}$ (rs2032582) and 3435C > T (rs1045642). They are associated with decreased efflux and possibly higher efficiency and toxicity. Two meta-analysis showed correlation between these polymorphisms and increased CR and OS, predominantly in Caucasians (10). The ABCB1 can be modulated with calcineurin antagonists, tyrosine kinase inhibitors, $\mathrm{H} 2$ receptor blockers, statins and calcium channel blockers (5).
Several studies analysing combination of anthracycline based chemotherapy with cyclosporine A, reported no impact on OS, but higher toxicity (5). Some studies evaluated toxicity related to $A B C B 1$ SNPs but the result was not conclusive. In addition, $A B C C 1$ polymorphisms have been associated with increased anthracycline-induced cardiotoxicity. However, $A B C C 3$ polymorphisms were associated with lower DFS while variations in ABCG2 gene correlated to longer OS and increased occurrence risk grade $\geq 3$ toxicities (7).

Several anthracycline influx pumps were also studied. The most evaluated is the SLCO1B1 (solute carrier organic anion transporter family member) with variant allele $\mathrm{C}$ of $521 \mathrm{~T}>\mathrm{C}$ (rs4149056) which is associated with hepatotoxicity in AML patients (7). Variants in other influx transporters, like human solute carrier SLC22A16, did not show clinical impact. However, wild types of SLC28A3, SLC25A37 and SLC22A2 polymorphisms have been associated with decreased DFS, but Bonferroni correction didn't confirm these associations (7).

\section{Anthracycline metabolising proteins}

Anthracyclines are metabolised by three metabolic ways: reduction of two electrons (the main way), reduction of one electron and deglycolisation. However, $50 \%$ of anthracyclines are eliminated from the body unchanged. The NADPH dependent cytosolic enzymes carbonyl reductase (CBR) and aldo-keto reductases (AKR) catalyse conversion of anthracyclines to a secondary alcohol, by reduction of two electrons. Variant alleles in CBR1 gene were associated with slower anthracycline metabolism and higher potential for cardiotoxicity, while variants in $C B R 3$ gene were associated with increased anthracycline clearance. Another metabolising way is anthracycline conversion to semiquinone radical, catalysed by oxidoreductases: cytosolic $\mathrm{NAD}(\mathrm{P}) \mathrm{H}$ quinone oxidoreductase (NQO1), mitochondrial NADH-ubiquinone oxidoreductase (NDUFS), xanthine dehydrogenase $(\mathrm{XDH})$ and nitric oxide (NO) synthetases (NOS1-3). Addressing this issue, variant allele $609 \mathrm{C}>\mathrm{T}$ in NQO1 is associated with lower CR rate, without influencing OS. The NOS3 expression was associated with favourable prognosis after chemotherapy in AML, possibly due to high production of NO, which induces apoptosis in leukemic cells. In a study of 225 intermediate risk Chinese AML patients with intermediate risk AML, NOS3 rs1799983 wild type carriers had increased mRNA expression of NOS3 and increased OS (7).

Polymorphisms of enzymes, not directly involved in anthracycline metabolism, have been evaluated in AML, too. Namely, $C Y P 1 A 1^{*} 2 A$ polymorphism (rs4646903) was related to lower OS, while SNP rs2070673 of CYP2E1 was associated with liver toxicity. Variants of P450 oxidoreductases $(P O R)$, that catalyse one electron reduction of anthracyclines, are associated with cardiotoxicity (rs2868177, rs13240755 and rs4732513) $(7,11)$. The alcohol dehydrogenase $(\mathrm{ADH})$ metabolises ethanol conversion to aldehyde, that is either converted by aldehyde dehydrogenase to 
acetate or which is accumulated intracellularly in form of DNA and protein adducts (7). Variants in both enzymes result in abnormal aldehyde accumulation. Minor alleles of $A D H$ (rs1826909 and rs6811453 in high linkage disequilibrium) were associated with decreased CR after induction, including ara-C, idarubicin, fludarabine and gentuzumab ozogamicin (7).

Cellular antioxidants - Glutathione S-transferases (GST)

One of the most important cellular antioxidants are GST. Their isoenzymes catalase transfer of sulfhydryl groups (-SH) from reduced form of glutathione to many endogenous and exogenous substrates, including anthracyclines $(5,7)$. GST oppose to ROS is created by anthracyclines. Seven classes constitute cytosolic GST family, where alpha $(\alpha)$, theta $(\tau), \mathrm{mu}(\mu)$ and pi $(\pi)$, are the most studied in humans. Polymorphisms GSTT1 and GSTM1 were linked to the development of a variety of malignancies, including AML (5). Homozygous deletion resulting in null genotypes of GSTT1 and GSTM1, with absent enzyme activity, were associated with lower CR rate after induction chemotherapy and decreased OS (5). A meta-analysis of null genotype of GSTT1 and double null genotypes of GSTT1 and GSTM1 were associated with reduced response after induction cycle, lower progression free survival (PFS) and shorter OS in AML patients, especially in Asians (12). Other GST polymorphisms, of GSTM1 and GSTT1 were correlated with lower DFS. In addition, SNP

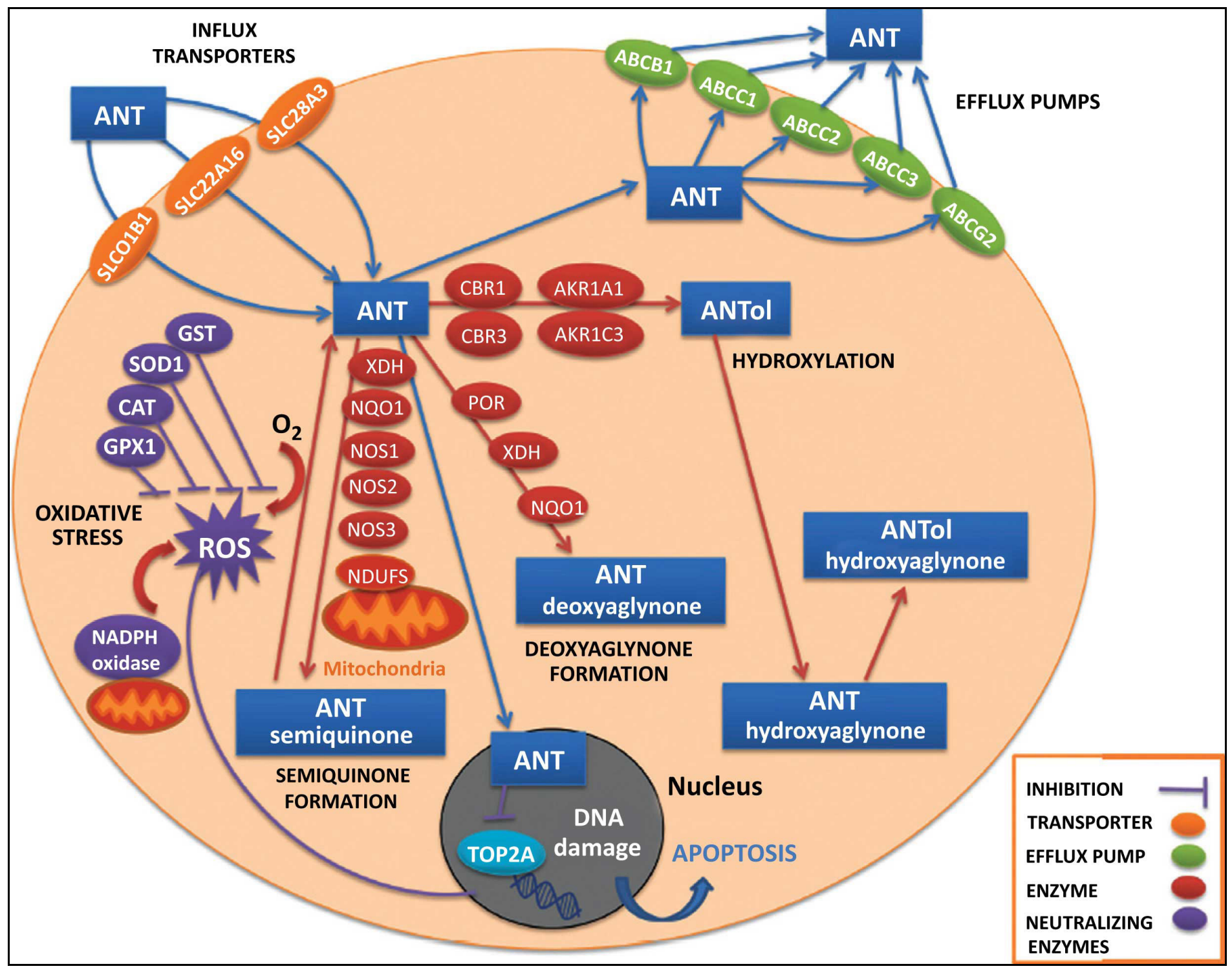

Figure 2. Main candidate genes involed in anthracycline metabolism (adopded from Megias-Vericat et al)

Abbreviations: ANT (anthracycline), ROS (reactive oxygene species), ABC (ATP binding casette efflux transporters), CBR (carbonyl reductase), AKR (aldo-keto reductases), ANTol (anthracycline alcohol), POR (cytohrome P450), $\mathrm{XDH}$ (xantine dehidrogenase), NQO (NAD(P)H quinone dehydrogenase), NOS (nitric oxide syntethase), NDUFS (NADH-ubiquinone oxidoreductase), GST (glutathion S-transferase), SOD (superoxide dismutase), CAT (catalaze), GPX (glutathione peroxidase), SLCO (solute carrier organic anion influx transporter), SLC (human solute carrier influx transporter) TOP2A (topoisomerase II) 
rs1695 of GSTP1, resulting in less active enzyme and reduced anthracycline clearance, was correlated with higher OS, and less hematologic toxicity, however, other studies did not confirm these association (13).

Effects of other antioxidative enzymes polymorphisms and clinical outcome were also studied. Sulfotransferase (SULT) catalyses sulphate conjugation of many compounds, including anthracyclines. In AML, SULT polymorphism rs1402467 in homozygous state (CC or GG genotype) demonstrated higher RR and decreased DFS. Polymorphism (rs4880) of Mangan superoxide dismutase (MnSOD), that eliminates superoxide, were located outside the mitochondria and showed less potency. Presence of this variant was associated with increased OS and increased mortality, as a result of decreased mitochondrial antioxidative capacity. However, SNPs in Catalase $(C A T)$ and Glutathione peroxidase (GP) did not influence clinical outcome. Furthermore, NADPH oxidase catalyses the ROS generation that leads to cell damage and anthracycline-induced cardiotoxicity (13). Effect of NADPH oxidase subunits polymorphisms in Caucasians showed that minor alleles of RAC2 (rs13058338) and NCF4 (rs1883112) subunits are associated with increased CR. In contrast, homozygous polymorphism of CYBA subunit (rs4673) is related to decreased OS. Furthermore, homozygous polymorphism of NCF and heterozygous CYBA genotype were related to anthracycline-induced cardiotoxicity (13). Figure 2 represents key enzymes and transporters in anthracycline pathway.

\section{Regulation of apoptosis in AML}

Alongside of disruption of cellular pathways vital for cell survival, an important effect of chemotherapeutics in elimination of malignant cells is a promotion of programmed cell death (apoptosis) (14). There are two ways of apoptosis induction: extrinsic, primarily regulating the cell turnover and mutant cell elimination and the intrinsic way, responsible for chemotherapy effect. Namely, double strand DNA brakes build up after chemotherapy and initiate intrinsic way. Proapoptotic Bax protein /B-cell lymphoma protein 2 (Bcl-2)-associated $\mathrm{X}(\mathrm{Bax}) /$ initiate stepwise reactions that lead to release of cytochrome $\mathrm{C}$ from mitochondria, activation of caspases and finally, cell death. Conversely, antiapoptotic $\mathrm{Bcl}-2$ protein prevents Bax from releasing cytochrome $C$, with consequent cell survival. Fragmentation of DNA supresses $B c l-2$ and induces Bax trough p53 (14).

Apoptotic deregulation in AML, as one of the MDR mechanisms, has been evaluated recently (14). Namely, it is believed that increased Bax and decreased $\mathrm{Bcl}-2$ expressions together with high $\mathrm{Bax} / \mathrm{Bcl}-2$ ratio are related to high CR rate and longer OS, due to favoured apoptosis. They are regarded as good prognostic markers, but the studies reported conflicting results on this topic. Some studies showed that high CR rate is in correlation with high Bax expression, considering Bax a good pro-apoptotic indicator in AML (15). In contrast, some studies showed that increased Bax expression was correlated with decreased DFS and OS $(16,17)$, while the other showed no impact on clinical outcomes (14). Conversely, elevated expression of $\mathrm{Bcl}-2$ was in correlation with increased $\mathrm{RR}$, but did not affect outcome (18). Another study reported association of increased $\mathrm{Bcl}-2$ expression with longer median OS and $\mathrm{CR}$ duration in the poor karyotype risk group, while a shorter median OS in intermediate and favourable karyotype risk group of AML patients (19). Concerning Bax/Bcl-2 ratio studies reported that high ratio was correlated with increased $\mathrm{CR}$ rate. Conversely, low $\mathrm{Bax} / \mathrm{Bcl}-2$ ratio was linked to less differentiated cell types (AML-M0 or M1), poor risk cytogenetics (20) and lower OS (17). Addressing this issue, Bcl-2 being a target for $\mathrm{Bcl}-2$ inhibitor venetoclax, sole or in combination with hypomethylating agents or low dose ara-C shows good results in unfit elderly AML patients (21).

\section{Conclusions and future perspectives}

The use of pre-treatment pharmacogenetic information in tailoring AML treatment has only just started to develop. In this review, we have summarized results from studies that investigated how genetic variability in the genes involved in ara- $\mathrm{C}$ and anthracycline metabolic pathways, together with genes that regulate apoptosis, affects therapy response and survival in AML patients. Findings from these studies can help us to identify patients who will benefit from standard AML therapy. Moreover, new genes and gene variations discovered in these studies can be used as targets for novel AML drugs.

Literature

1. Hatipoglu OF, Bender O, Gunduz E, Gunduz M. Pharmacogenomics in Acute Myeloid Leukemia. In: Barh D, Dhawan D, Ganguly N, editors. Omics for Personalized Medicine. New Delhi: Springer; 2013. p.237-48.

2. Döhner H, Estey E, Grimwade D, et al. Diagnosis and management of AML in adults: 2017 ELN recommendations from an international expert panel. Blood 2017;129(4):424-47.

3. Maksimovic N, Zaric M, Gazibara T, et al. Incidence and Mortality Patterns of Acute Myeloid Leukemia in Belgrade, Serbia (19992013). Medicina (Kaunas) 2018;54(1):5.

4. Isidori A, Loscocco F, Curti A, Amadori S, Visani G. Genomic profiling and predicting treatment response in acute myeloid leukemia. Pharmacogenomics. 2019;20(7):467-470.

5. Emadi A, Karp JE. The clinically relevant pharmacogenomic changes in acute myelogenous leukemia. Pharmacogenomics 2012;13(11):1257-69.

6. Mlakar V, Huezo-Diaz Curtis P, Satyanarayana Uppugunduri CR, et al. Pharmacogenomics in Pediatric Oncology: Review of Gene-Drug Associations for Clinical Use. Int J Mol Sci 2016;17(9):1502.

7. Megías-Vericat JE, Montesinos P, Herrero MJ, et al. Pharmacogenomics and the treatment of acute myeloid leukemia. Pharmacogenomics 2016;17(11):1245-72.

8. Lamba JK. Genetic factors influencing cytarabine therapy. Pharmacogenomics 2009; 10(10):1657-74.

9. Wan H, Zhu J, Chen F, et al. SLC29A1 single nucleotide polymorphisms as independent prognostic predictors for survival of patients with acute myeloid leukemia: an in vitro study. J Exp Clin Cancer Res. 2014;33(1):90. 
10. Megías-Vericat JE, Rojas L, Herrero MJ, et al. Influence ofABCB1 polymorphisms upon the effectiveness of standard treatment for acute myeloid leukemia: a systematic review and meta-analysis of observational studies. Pharmacogenomics 2015;15:109-118.

11. Lubieniecka JM, Graham J, Heffner D et al. A discovery study of daunorubicin induced cardiotoxicity in a sample of acute myeloid leukemia patients prioritizes $\mathrm{P} 450$ oxidoreductase polymorphisms as a potential risk factor. Front Genet 2013;4:231.

12. Xiao Q, Deng D, Li H, et al. GSTT1 and GSTM1 polymorphisms predict treatment outcome for acute myeloid leukemia: a systematic review and meta-analysis. Ann Hematol. 2014;93(8):1381-90.

13. Megias-Vericat JE, Martinez-Cuadron D, Herrero MJ, et al. Pharmacogenetics of Metabolic Genes of Anthracyclines in Acute Myeloid Leukemia. Curr Drug Metab 2018;19(1):55-74.

14. Kulsoom B, Shamsi TS, Afsar NA, et al. Bax, Bcl-2, and Bax/Bcl-2 as prognostic markers in acute myeloid leukemia: are we ready for Bcl2-directed therapy? Cancer Manag Res 2018; 10:403-16.

15. Prokop A, Wieder T, Sturn I, et al. Relapse in childhood acute lymphoblastic leukemia is associated with a decrease of the Bax/Bcl-2 ratio and loss of spontaneous caspase-3 processing in vivo. Leukemia. 2000;14:1606-13.
16. Hogarth LA, Hall AG. Increased BAX expression is associated with an increased risk of relapse in childhood acute lymphocytic leukemia. Blood. 1999;93:2671-8.

17. Köhler T, Schill C, Deininger MW, et al. High Bad and Bax mRNA expression correlate with negative outcome in acute myeloid leukemia (AML). Leukemia. 2002;16:22-9.

18. Aref S, Salama O, Al-Tonbary Y, Mansour A. Assessment of bcl-2 expression as modulator of fas mediated apoptosis in acute leukemia. Hematology. 2004;9:113-21.

19. Kornblau SM, Thall PF, Estrov Z, et al. The prognostic impact of BCL2 protein expression in acute myelogenous leukemia varies with cytogenetics. Clin Cancer Res. 1999; 5:1758-66.

20. Del Poeta G, Venditti A, Del Principe MI, et al. Amount of spontaneous apoptosis detected by $\mathrm{Bax} / \mathrm{Bcl}-2$ ratio predicts outcome in acute myeloid leukemia (AML). Blood. 2003;101:2125-31.

21. Guerra VA, DiNardo C, Konopleva M. Venetoclax-based Therapies for Acute Myeloid Leukemia. BestPract Res Clin Haematol. 2019;32(2):145-153. 